\title{
Aquaporin 1 Is an Independent Marker of Poor Prognosis in Lung Adenocarcinoma
}

\author{
Sumi Yun ${ }^{1,2 *} \cdot$ Ping-Li Sun ${ }^{3 *}$ \\ Yan $\mathrm{Jin}^{4} \cdot$ Hyojin $\mathrm{Kim}^{1}$ \\ Eunhyang Park ${ }^{1}$. Soo Young Park ${ }^{1}$ \\ Kyuho Lee ${ }^{1} \cdot$ Kyoungyul Lee ${ }^{5}$ \\ Jin-Haeng Chung ${ }^{1}$
}

'Department of Pathology, Seoul National University Bundang Hospital, Seongnam; 2Department of Pathology, Soonchunhyang University Hospital, Seoul, Korea; ${ }^{3}$ Department of Pathology, Second Hospital of Jilin University, Changchun; ${ }^{\circledR}$ Department of Pathology, Fudan University, Shanghai Cancer Center, Shanghai, China; ${ }^{5}$ Department of Pathology, Kangwon National University Hospital,

Chuncheon, Korea

\section{Received: February 15, 2016 \\ Revised: March 27, 2016}

Accepted: March 29, 2016

\author{
Corresponding Author \\ Jin-Haeng Chung, MD, PhD \\ Department of Pathology, Seoul National University \\ Bundang Hospital, 82 Gumi-ro 173beon-gil, \\ Bundang-gu, Seongnam 13620, Korea \\ Tel: $+82-31-787-7713$ \\ Fax: +82-31-787-4012 \\ E-mail: chungjh@snu.ac.kr \\ *Sumi Yun and Ping-Li Sun contributed equally to \\ this work.
}

\begin{abstract}
Background: Aquaporin 1 (AQP1) overexpression has been shown to be associated with uncontrolled cell replication, invasion, migration, and tumor metastasis. We aimed to evaluate AQP1 expression in lung adenocarcinomas and to examine its association with clinicopathological features and prognostic significance. We also investigated the association between AQP1 overexpression and epithelial-mesenchymal transition (EMT) markers. Methods: We examined AQP1 expression in 505 cases of surgically resected lung adenocarcinomas acquired at the Seoul National University Bundang Hospital from 2003 to 2012. Expression of AQP1 and EMT-related markers, including Ecadherin and vimentin, were analyzed by immunohistochemistry and tissue microarray. Results: AQP1 overexpression was associated with several aggressive pathological parameters, including venous invasion, lymphatic invasion, and tumor recurrence. AQP1 overexpression tended to be associated with higher histological grade, advanced pathological stage, and anaplastic lymphoma kinase (ALK) translocation; however, these differences were not statistically significant. In addition, AQP1 overexpression positively correlated with loss of $\mathrm{E}$-cadherin expression and acquired expression of vimentin. Lung adenocarcinoma patients with AQP1 overexpression showed shorter progression-free survival (PFS, 46.1 months vs. 56.2 months) compared to patients without AQP1 overexpression. Multivariate analysis confirmed that AQP1 overexpression was significantly associated with shorter PFS (hazard ratio, 1.429; 95\% confidence interval, 1.033 to $1.977 ; p=.031$ ). Conclusions: AQP1 overexpression was thereby concluded to be an independent factor of poor prognosis associated with shorter PFS in lung adenocarcinoma. These results suggested that AQP1 overexpression might be considered as a prognostic biomarker of lung adenocarcinoma.
\end{abstract}

Key Words: Aquaporin 1; Adenocarcinoma; Tissue array analysis; Invasion; Epithelial-mesenchymal transition
Non-small cell lung cancer (NSCLC) is one of the major causes of cancer-related deaths, and the treatment of NSCLC remains challenging. Adenocarcinoma comprises more than half of all NSCLC. Recent research has yielded a better understanding of the carcinogenesis, molecular subtypes, and prognostic factors of NSCLC, and several novel agents targeting oncogenic pathways have been introduced for the clinical treatment of NSCLC. Despite advancements in surgical treatment, selective targeted therapy, and early cancer detection, the relative long-term survival rate of NSCLC is still lower than that of other malignancies. $^{1-3}$ Therefore, a better understanding of the mechanism(s) of NSCLC tumor progression is needed, and useful prognostic biomolecular markers for accurate prediction of the clinical outcome of NSCLC are of great clinical significance.

Aquaproin 1 (AQP1) is a member of the aquaporin family found in cell membranes, and primarily facilitates transcellular water transport. AQP1 is expressed in various tissues such as kidney, choroid plexus, and gall bladder, and has a role in various physiological and pathological processes. ${ }^{4-6}$ Recently, several reports have revealed that AQP1 overexpression is associated with uncontrolled cell replication, invasion, migration, and metastasis in tumors. ${ }^{5,7}$ In lung cancer, various studies have shown the preferential expression of AQP1 in adenocarcinoma, and an association between AQP1 expression and tumor cell differenti- 
ation, invasion, metastasis, and poor prognosis. ${ }^{8-12}$ However, the molecular role and mechanism of AQP1 in cancer cell invasion remains unclear.

Epithelial-mesenchymal transition (EMT) is a complex process in which epithelial cells lose cell-cell adhesions and gain migratory properties. EMT has been implicated in many aspects of cancer, such as tumor cell invasion, metastasis, drug resistance, and poor clinical outcome. The mechanism of EMT results from complex signaling pathway crosstalk between tumor cells and the tumor microenvironment. Several studies have shown that alterations in EMT-related markers are observed in lung adenocarcinoma, and these alterations enhanced tumor progression and metastasis. ${ }^{13-15}$ Recent results have indicated that AQP1 overexpression is correlated to EMT in both colon and breast cancers, ${ }^{16-18}$ but the correlation has not yet been investigated in lung cancer.

In the present study, we analyzed the immunohistochemical expression of AQP1 in a large series of lung adenocarcinoma specimens, and evaluated the frequency and pattern of AQP1 expression along with its association with clinicopathological factors, molecular subtypes, and patient outcome. In addition, we explored the correlation between AQP1 overexpression and alteration of the EMT-related markers E-cadherin and vimentin.

\section{MATERIALS AND METHODS}

\section{Tissue samples and classification}

Tumor samples were collected from 505 consecutive lung adenocarcinoma patients who underwent curative surgical resection at Seoul National University Bundang Hospital between May 2003 and December 2012. Clinicopathological information and follow-up data were obtained by reviewing the medical and the pathological records of the enrolled patients. Smoking history was defined as smokers who have smoked 100 cigarettes, and never-smokers who have never smoked or smoked less than 100 cigarettes in their life time. Two pathologists (S.Y. and J.-H. C) independently reviewed the hematoxylin and eosin stained slides and classified the diagnosis according to the International Association for the Study of Lung Cancer/American Thoracic Society/European Respiratory Society (IASLC/ATS/ERS) criteria and the 2015 World Health Organization (WHO) classification system. ${ }^{19,20}$ Histological grade was based on the predominant histological subtypes as follows: grade 1, lepidic; grade 2, acinar and papillary; and grade 3 , solid and micropapillary. Tumors were staged according to the American Joint Committee on Cancer, seventh staging system. Progression-free survival (PFS) was measured from the date of surgery until disease progression or death. Overall survival (OS) was measured from the date of surgery to the time of death or last follow-up visit. The Institutional Review Board of the Seoul National University Bundang Hospital approved this study.

\section{Tissue microarray}

The most representative areas were obtained for each tumor sample and arranged for tissue microarray (TMA). Tissue cores with a diameter of $2 \mathrm{~mm}$ were embedded within TMA blocks, which were sectioned into series of $4-\mu \mathrm{m}$-thick slices and then stained with hematoxylin and eosin; immunohistochemical labeling was then performed.

\section{Immunohistochemical staining and assessment}

Immunohistochemistry for AQP1, E-cadherin, and vimentin were performed according to the antibody manufacturer's instructions. The following antibodies were used: AQP1 (B-11, 1:100, Santa Cruz Biotechnology, Santa Cruz, CA, USA), Ecadherin (SPM471, 1:150, Thermo Fisher Scientific, Carlsbad, CA, USA), and vimentin (1:100, 4A4, Zeta Corporation, Arcadia, CA, USA). AQP1 showed both apicolateral staining and complete circumferential membranous staining (Fig. 1). AQP1 overexpression was defined when $\geq 25 \%$ showed membranous staining with loss of polarization, as previously reported. ${ }^{21} \mathrm{Im}-$ munohistochemical stains of E-cadherin and vimentin were scored using a semi-quantitative evaluation for each case. The intensity of staining was scored on a four-point scale as follows: 0 , no staining; 1 , weak staining; 2 , moderate staining; and 3, strong staining. The score was based on the fraction of positive cells $(1 \%-100 \%)$. The final score was calculated by multiplying the intensity score to the fraction score, generating a total range of 0-300. Scores of 0-100 and 101-300 were considered as negative and positive, respectively, as previously reported. ${ }^{22}$

\section{Molecular characteristics}

Translocation of anaplastic large cell lymphoma kinase ( $A L K)$ was evaluated in 440 cases by fluorescence in situ hybridization analysis using a probe to ALK (Vysis LSI ALK dual color, breakapart rearrangement probe, Abbott Molecular, Des Plaines, IL, USA); translocation was observed in 28 out of 440 cases (6.4\%). Epidermal growth factor receptor (EGFR) mutations of exon 18 to 21 and KRAS mutations of codon 12 and 13 were evaluated in 484 and 413 cases using polymerase chain reaction and direct DNA sequencing, as previously described. ${ }^{23}$ EGFR and KRAS mutations were identified in $49.0 \%$ (237/484) and 6.1\% 

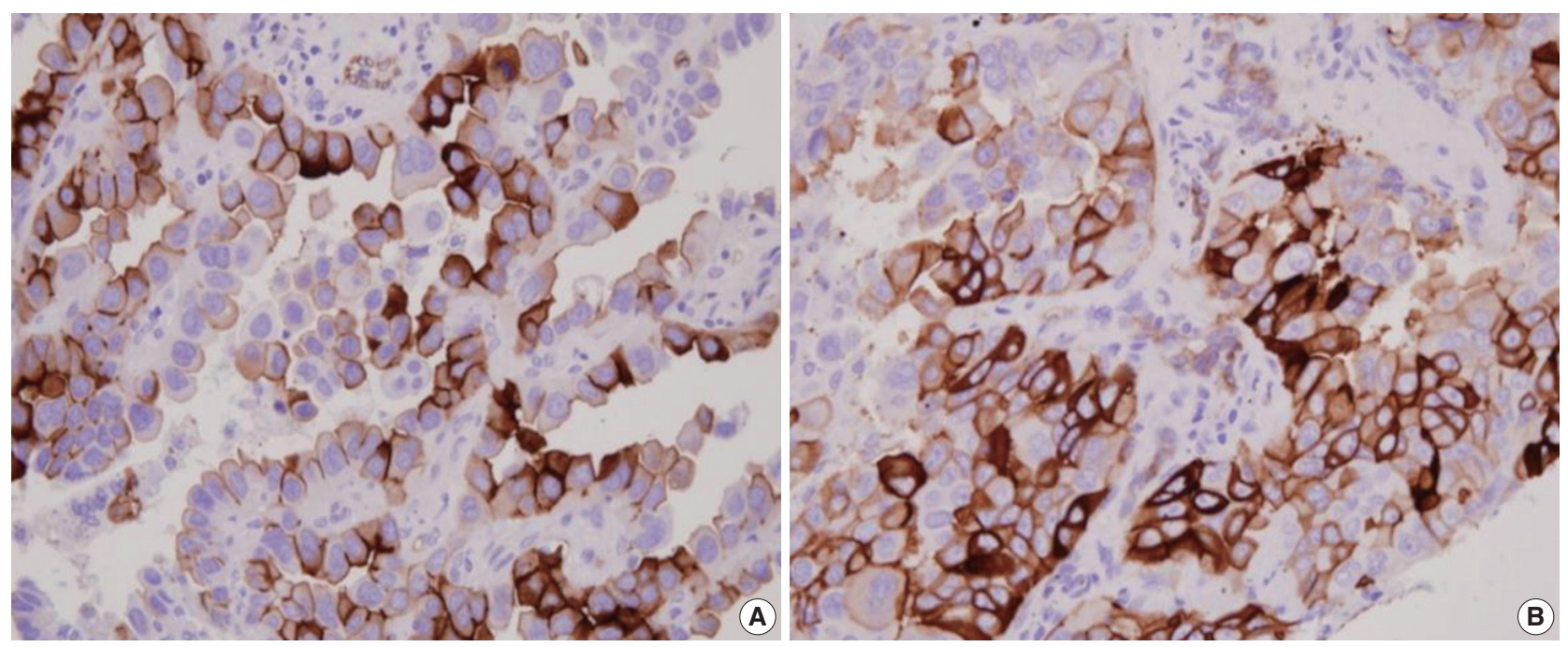

Fig. 1. Immunohistochemical expression of aquaporin 1 in lung adenocarcinomas. (A) Apicolateral pattern. (B) Circumferential membranous staining.

(25/413) of cases, respectively.

\section{Statistical analysis}

All statistical analyses were performed using SPSS ver. 18.0 (SPSS Inc., Chicago, IL, USA). The association between immunohistochemistry results and clinicopathological variables was assessed by chi-square test, Fisher exact test, or Spearman's rank correlation test. Kaplan-Meier analysis with log-rank test and multivariate cox regression analysis were performed for survival analysis. Statistical significance was defined as $\mathrm{p}<.05$.

\section{RESULTS}

\section{Clinicopathological characteristics}

The clinicopathological characteristics of patients are summarized in Table 1 . The tumor specimens in this study were from 505 lung adenocarcinoma patients, consisting of 247 male (48.9\%) and 258 female (51.1\%) patients. The mean age of patients was 62.9 years (range, 21 to 83 years), with 302 nonsmokers (59.8\%) and 203 smokers (40.2\%). With respect to tumor pathology, 274 samples (54.3\%) were pathologic stage I, $93(18.4 \%)$ were stage II, 109 (21.6\%) were stage III, and 29 $(5.7 \%)$ were stage IV. According to the new IASLC/ATS/ERS adenocarcinoma classification and the 2015 WHO classification, 298 (59.0\%) were acinar, 83 (16.4\%) were papillary, 73 $(14.5 \%)$ were solid, $33(6.5 \%)$ were lepidic, 8 (1.6\%) were micropapillary, and $10(2.0 \%)$ were invasive mucinous subtypes. Venous invasion, lymphatic invasion, and perineural invasion were observed in $25.7 \%$ (130/505), $50.1 \%$ (253/505), and $7.1 \%$
(36/505) of samples, respectively.

\section{AQP1 protein expression by immunohistochemistry}

AQP1 expression was observed in the vascular endothelial cells and the apicolateral surfaces of hyperplastic type II pneumocytes around tumors. AQP1 was also detected in myoepithelial cells of bronchial glands and red blood cells (data not shown).

\section{Association of AQP1 overexpression with clinicopathological features}

AQP1 overexpression (Fig. 1) was detected in $20.8 \%$ of adenocarcinoma cases (105/505). Table 1 shows the association of AQP1 overexpression with clinicopathological variables. There was a significant association of $\mathrm{AQP1}$ overexpression with venous invasion $(\mathrm{p}=.035)$ and lymphatic invasion $(\mathrm{p}=.039)$. The recurrence rate of patients with AQP1 overexpression was significantly higher than that of patients without AQP1 overexpression $(\mathrm{p}=.029)$. AQP1 overexpression was not associated with higher histological grade $(\mathrm{p}=.097)$, pleural invasion $(\mathrm{p}=.131)$, and other clinicopathological variables or molecular characteristics, such as EGFR and KRAS mutation and ALK rearrangement.

\section{Association between AQP1 overexpression and EMT-related marker expression}

In total, immunohistochemical analyses of E-cadherin and vimentin were performed for 479 and 471 cases, respectively. Loss of E-cadherin expression was observed in 201 of 479 cases (42.0\%), and expression of vimentin was observed in 192 of 471 cases (40.8\%). We compared the association of AQP1 over- 
Table 1. Clinicopathological characteristics of AQP1 overexpression in 505 lung adenocarcinoma patients

\begin{tabular}{|c|c|c|c|c|}
\hline \multirow{2}{*}{ Variable } & \multirow{2}{*}{ Total } & \multicolumn{3}{|c|}{ AQP1 overexpression } \\
\hline & & Negative & Positive & $p$-value \\
\hline Sex & & & & .938 \\
\hline Male & 247 & $196(79.4)$ & $51(20.6)$ & \\
\hline Female & 258 & $204(79.1)$ & $54(20.9)$ & \\
\hline Age (yr) & & & & .092 \\
\hline$\leq 60$ & 181 & $136(75.1)$ & 45 (24.9) & \\
\hline$>60$ & 324 & $264(81.5)$ & $60(18.5)$ & \\
\hline Smoking history & & & & .347 \\
\hline Non-smoker & 302 & $235(77.8)$ & 67 (22.2) & \\
\hline Smoker & 203 & $165(81.3)$ & $38(18.7)$ & \\
\hline Tumor size (cm) & & & & .145 \\
\hline$\leq 3$ & 276 & $212(76.8)$ & 64 (23.2) & \\
\hline$>3$ & 229 & $188(82.1)$ & 41 (17.9) & \\
\hline Histological grade & & & & .097 \\
\hline G1 & 34 & 30 (88.2) & $4(11.8)$ & \\
\hline G2 & 390 & $312(80.0)$ & $78(20.0)$ & \\
\hline G3 & 81 & 58 (71.6) & $23(28.4)$ & \\
\hline Acinar predominant & & & & .368 \\
\hline No & 207 & $168(81.2)$ & 39 (18.8) & \\
\hline Yes & 298 & $232(77.9)$ & $66(22.1)$ & \\
\hline Papillary predominant & & & & .120 \\
\hline No & 422 & $339(78.0)$ & $93(22.0)$ & \\
\hline Yes & 83 & $71(85.5)$ & $12(14.5)$ & \\
\hline Lepidic predominant & & & & .087 \\
\hline No & 472 & $370(78.4)$ & $102(21.6)$ & \\
\hline Yes & 33 & 30 (90.9) & $3(9.1)$ & \\
\hline Solid predominant & & & & .233 \\
\hline No & 432 & $346(80.1)$ & 86 (19.9) & \\
\hline Yes & 73 & $54(74.0)$ & $19(26.0)$ & \\
\hline Others $^{\mathrm{a}}$ & & & & .552 \\
\hline No & 487 & 387 (79.5) & $100(20.5)$ & \\
\hline Yes & 18 & 13 (72.2) & $5(27.8)$ & \\
\hline Pleural invasion & & & & .131 \\
\hline Absent & 283 & $231(81.6)$ & $52(18.4)$ & \\
\hline Present & 222 & $169(76.1)$ & $53(23.9)$ & \\
\hline Venous invasion & & & & $.035^{\mathrm{b}}$ \\
\hline Absent & 377 & 307 (81.4) & 70 (18.6) & \\
\hline Present & 130 & 93 (72.7) & 35 (27.3) & \\
\hline Lymphatic invasion & & & & $.039^{b}$ \\
\hline Absent & 252 & 209 (82.9) & $43(17.1)$ & \\
\hline Present & 253 & $193(75.5)$ & $62(24.5)$ & \\
\hline Perineural invasion & & & & .290 \\
\hline Absent & 469 & $369(78.7)$ & $100(21.2)$ & \\
\hline Present & 36 & 31 (86.1) & $5(13.9)$ & \\
\hline pTNM stage & & & & .072 \\
\hline $\mid-\|$ & 367 & $298(81.2)$ & 69 (18.8) & \\
\hline III-IV & 138 & $102(73.9)$ & 36 (26.1) & \\
\hline Recurrence & & & & $.029^{b}$ \\
\hline No & 311 & 256 (82.3) & $55(17.7)$ & \\
\hline Yes & 194 & $144(74.2)$ & $50(25.8)$ & \\
\hline Death & & & & .130 \\
\hline No & 415 & $334(80.5)$ & 81 (19.5) & \\
\hline
\end{tabular}

\begin{tabular}{lrrrr}
\hline \multirow{2}{*}{ Variable } & \multirow{4}{c}{ Total } & \multicolumn{3}{c}{ AQ1 overexpression } \\
\cline { 3 - 5 } & & Negative & Positive & p-value \\
\hline Yes & 90 & $66(73.3)$ & $24(26.7)$ & \\
EGFR mutation $(n=484)$ & & & & .583 \\
$\quad$ Wild type & 247 & $193(78.1)$ & $54(21.9)$ & \\
$\quad$ Mutant type & 237 & $190(80.2)$ & $47(19.8)$ & \\
KRAS mutation $(n=413)$ & & & & .326 \\
$\quad$ Wild type & 388 & $311(80.2)$ & $77(19.8)$ & \\
$\quad$ Mutant type & 25 & $18(72.0)$ & $7(28.0)$ & \\
ALK translocation & & & & .089 \\
$\begin{array}{l}(n=440) \\
\quad \text { Wild type }\end{array}$ & 412 & $334(81.1)$ & $79(18.9)$ & \\
$\quad$ Mutant type & 28 & $19(67.9)$ & $9(32.1)$ & \\
\hline
\end{tabular}

Values are presented as number (\%).

AQP1, aquaporin 1; EGFR, epidermal growth factor receptor; ALK, anaplastic lymphoma kinase.

anvasive mucinous and micropapillary subtype; bStatistically significant value.

expression to expression of E-cadherin or vimentin (Table 2) and found that AQP1 overexpression was correlated with loss of E-cadherin expression $(\mathrm{p}=.011)$ and expression of vimentin $(\mathrm{p}$ $<.001)$.

\section{Survival analysis according to AQP1 overexpression}

At the time of analysis, the median PFS was 31.0 months (range, 1 to 84 months) and the median OS was 39 months (range, 1 to 84 months). During this time, 194 patients (38.4\%) suffered tumor recurrence and 90 patients (17.8\%) died from cancer. Survival analysis using Kaplan-Meier and Cox proportional hazards analyses were performed to evaluate the prognostic impact of AQP1 overexpression. As shown in Fig. 2, Kaplan-Meier revealed that PFS of patients with AQP1 overexpression was significantly shorter than that of the patients without AQP1 overexpression group ( $\mathrm{p}=.018$ ). However, AQP1 overexpression had no prognostic impact on OS $(\mathrm{p}=.234)$. Univariate analysis indicated that larger tumor size $(\mathrm{p}<.001)$, higher histological grade $(\mathrm{p}=$ .032 ), pleural invasion ( $\mathrm{p}<.001$ ), venous invasion $(\mathrm{p}<.001)$, lymphatic invasion $(\mathrm{p}<.001)$, perineural invasion $(\mathrm{p}=.043)$, $\mathrm{pTNM}$ stage ( $\mathrm{p}<.001$ ), and AQP1 overexpression (46.1 months vs. 56.2 months, $\mathrm{p}=.020$ ) were associated with shorter PFS (Fig. 2A). Multivariate cox regression analysis demonstrated AQP1 overexpression to be an independent factor indicating poor prognosis with regard to PFS in patients with lung adenocarcinoma (hazard ratio [HR], 1.429; 95\% confidence interval [CI], 1.033 to $1.977 ; \mathrm{p}=.031$ ). Larger tumor size (HR, 1.797; 95\% CI, 1.336 to $2.418 ; \mathrm{p}<.001$ ), pleural invasion (HR, 1.372; 95\% CI, 1.007 to $1.871 ; \mathrm{p}=.045)$, lymphatic invasion (HR, $1.547 ; 95 \% \mathrm{CI}$, 1.113 to $2.151 ; \mathrm{p}=.009)$, and pTNM stage (HR, $2.179 ; 95 \%$ CI, 1.586 to 2.995; $\mathrm{p}<.001$ ) were also independent prognostic 
Table 2. Correlation between AQP1 overexpression and EMT-related proteins

\begin{tabular}{|c|c|c|c|c|}
\hline \multirow{2}{*}{ Variable } & \multirow{2}{*}{ Total } & \multicolumn{3}{|c|}{ AQP1 overexpression } \\
\hline & & Negative & Positive & $p$-value \\
\hline E-Cadherin expression $(n=479)$ & & & & $.011^{\mathrm{a}}$ \\
\hline Decreased & 201 & $147(73.1)$ & $54(26.9)$ & \\
\hline Preserved & 278 & $230(82.7)$ & $48(17.3)$ & \\
\hline Vimentin expression $(n=471)$ & & & & $<.001^{\mathrm{a}}$ \\
\hline Negative & 279 & $238(85.3)$ & $41(14.7)$ & \\
\hline Positive & 192 & $133(69.3)$ & $59(30.7)$ & \\
\hline
\end{tabular}

Values are presented as number (\%).

$\mathrm{AQP1}$, aquaporin 1; EMT, epithelial-mesenchymal transition.

aStatistically significant value.
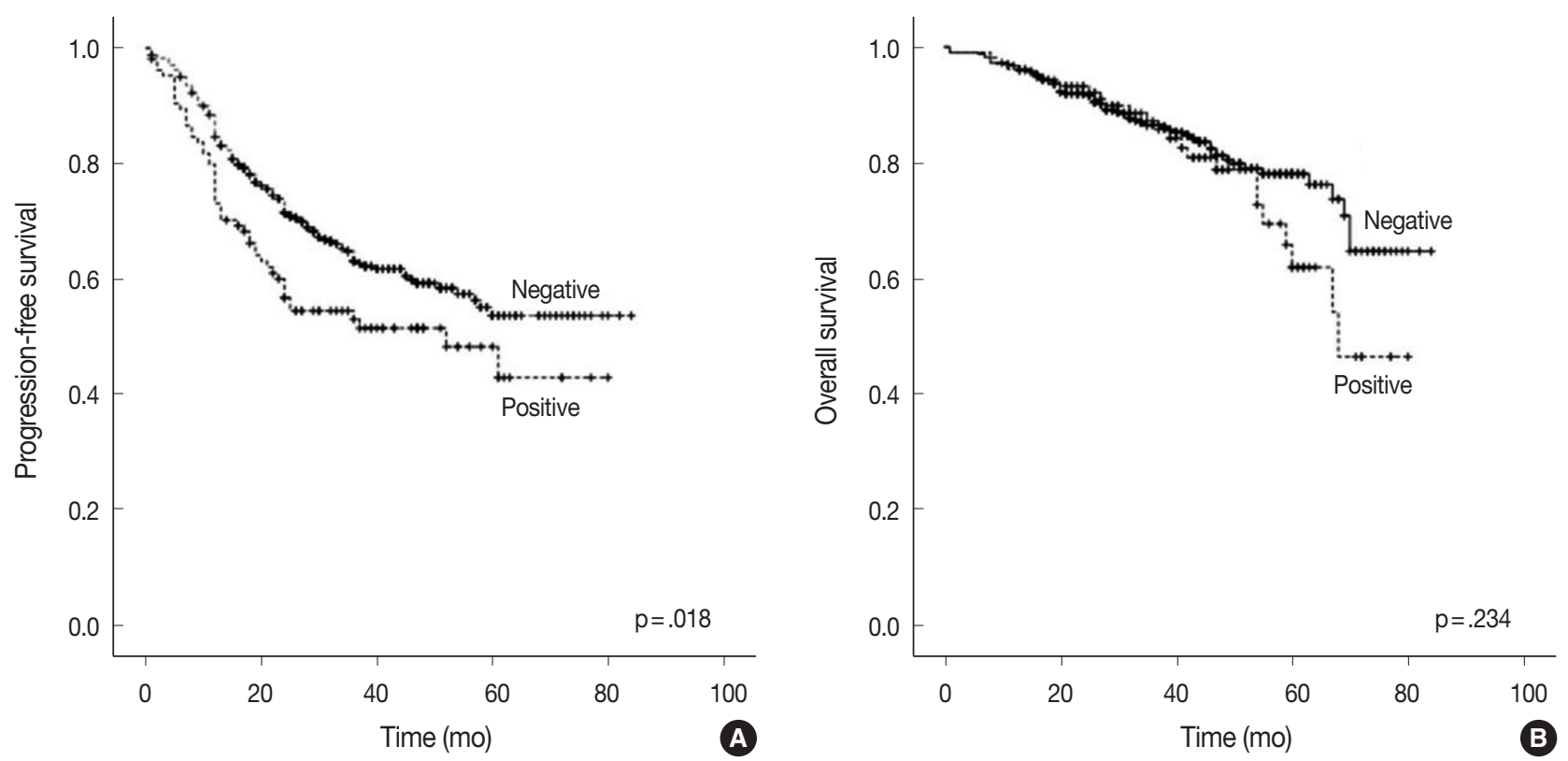

Fig. 2. Kaplan-Meier survival estimate graphs of progression-free survival (A) and overall survival (B) according to the overexpression of aquaporin 1 in lung adenocarcinoma patients.

factors associated with shorter PFS in lung adenocarcinoma. For OS, larger tumor size $(\mathrm{p}<.001)$, higher histological grade $(\mathrm{p}=$ $.021)$, pleural invasion $(\mathrm{p}<.001)$, venous invasion $(\mathrm{p}<.001)$, lymphatic invasion $(\mathrm{p}<.001)$, perineural invasion $(\mathrm{p}=.001)$, vimentin expression $(\mathrm{p}=.045)$, and pTNM stage $(\mathrm{p}<.001)$ reached statistical significance by univariate analysis. AQP1 overexpression was not associated with OS (63.2 months vs. 70.1 months, $\mathrm{p}=.237)$. In multivariate analysis, larger tumor size (HR, 1.775; 95\% CI, 1.137 to 2.771; $\mathrm{p}=.012$ ), venous invasion (HR, 2.129; 95\% CI, 1.352 to 3.354; $\mathrm{p}=.001$ ), and pTNM stage (HR, 4.789; $95 \% \mathrm{CI}, 3.026$ to $7.578 ; \mathrm{p}<.001)$ were statistically significant.

\section{DISCUSSION}

In the present study, we assessed the expression of AQP1 in
505 lung adenocarcinomas and evaluated the relationship between AQP1 overexpression and various clinicopathological factors and molecular characteristics, as well as the expression of EMT-related markers. Our study showed that AQP1 overexpression significantly correlated with several aggressive pathological factors and can be used as an independent prognostic factor for PFS in lung adenocarcinoma.

AQP1 is a plasma membrane channel involved in transepithelial water transport. ${ }^{5,7}$ Recently, the functional roles of AQP1 protein expression have been studied in various cancers. Previous studies have demonstrated that AQP1 is upregulated in several cancer tissues in vitro and in vivo, and AQP1 overexpression is associated with poor prognosis. ${ }^{11,13,16,17,24}$ Hoque et al. ${ }^{8}$ reported that upregulated AQP1 in lung cancer may play a role in cancer cell proliferation by resisting apoptosis. Machida et al. ${ }^{10}$ 
showed that AQP1 overexpression with a loss of polarization is associated with invasive growth and poor prognosis in lung adenocarcinomas. Consistent with previous observations, our study showed that AQP1 overexpression tended to be more frequently observed in the high grade histological subtypes of adenocarcinomas although it was not statistically significant.

We also analyzed the association of $A Q P 1$ overexpression with EMT-related markers (E-cadherin and vimentin). The loss of Ecadherin and increased expression of vimentin, both hallmarks of a mesenchymal phenotype, were frequently observed in tumors with $\mathrm{AQP1}$ overexpression. We hypothesized that AQP1 may participate in tumor progression through EMT (loss of E-cadherin and vimentin expression) in lung adenocarcinoma. EMT is implicated in tumor progression, invasion, metastasis, and poor prognosis in lung cancer. ${ }^{6,13,25}$ Recently, several reports have suggested that $\mathrm{AQP1}$ overexpression is associated with cancer cell invasion. AQP1 has been suggested to function as a linker molecule to promote EMT, or to stabilize the cytoskeletal complex. ${ }^{8,10,26,27}$ Similar results were reported in various cancers including colorectal cancer, breast cancer, and brain tumors, ${ }^{13,17,28}$ which is in line with our results. The exact biological and functional mechanism of AQP1 to promote EMT needed to be clarifiied by further studies. Of note, some studies demonstrated that tumor tissue showed intratumoral heterogeneity of AQP1 expression in brain tumor. ${ }^{24,29}$ Thus, it is necessary to clarify the intratumoral heterogenous distribution of AQP1 overexpression in cancer and its clinical significance.

In conclusion, we demonstrated that overexpression of AQP1 was significantly associated with venous invasion, lymphatic invasion, higher pathological stage, and cancer recurrence in lung adenocarcinomas. AQP1 was also deemed an independent marker of poor prognosis with regard to PFS. In particular, increased expression of AQP1 protein was associated with the expression of vimentin and loss of E-cadherin expression, suggesting that AQP1 overexpression may be involved in tumor cell invasion through facilitating EMT.

\section{Conflicts of Interest}

No potential conflict of interest relevant to this article was reported.

\section{Acknowledgments}

This work was supported by a grant of the Korean Health Technology R\&D Project, Ministry of Health \& Welfare, Republic of Korea (HI14C1907), the Basic Science Research Pro- gram through the National Research Foundation of Korea (NRF) funded by the Ministry of Education, Science and Technology (NRF-2013R1A1A2A10059757).

\section{REFERENCES}

1. Kim JE, Kim H, Choe JY, Sun P, Jheon S, Chung JH. High expression of Sonic hedgehog signaling proteins is related to the favorable outcome, EGFR mutation, and lepidic predominant subtype in primary lung adenocarcinoma. Ann Surg Oncol 2013; 20 Suppl 3: S570-6.

2. Seo AN, Yang JM, Kim H, et al. Clinicopathologic and prognostic significance of c-MYC copy number gain in lung adenocarcinomas. Br J Cancer 2014; 110: 2688-99.

3. Pao W, Girard N. New driver mutations in non-small-cell lung cancer. Lancet Oncol 2011; 12: 175-80.

4. Cuddapah VA, Sontheimer H. Ion channels and transporters [corrected] in cancer. 2. Ion channels and the control of cancer cell migration. Am J Physiol Cell Physiol 2011; 301: C541-9.

5. Verkman AS. More than just water channels: unexpected cellular roles of aquaporins. J Cell Sci 2005; 118(Pt 15): 3225-32.

6. Wang J, Feng L, Zhu Z, et al. Aquaporins as diagnostic and therapeutic targets in cancer: how far we are? J Transl Med 2015; $13: 96$.

7. Nico B, Ribatti D. Aquaporins in tumor growth and angiogenesis. Cancer Lett 2010; 294: 135-8.

8. Hoque MO, Soria JC, Woo J, et al. Aquaporin 1 is overexpressed in lung cancer and stimulates NIH-3T3 cell proliferation and anchorage-independent growth. Am J Pathol 2006; 168: 1345-53.

9. López-Campos JL, Sánchez Silva R, Gómez Izquierdo L, et al. Overexpression of aquaporin-1 in lung adenocarcinomas and pleural mesotheliomas. Histol Histopathol 2011; 26: 451-9.

10. Machida Y, Ueda Y, Shimasaki M, et al. Relationship of aquaporin 1 , 3 , and 5 expression in lung cancer cells to cellular differentiation, invasive growth, and metastasis potential. Hum Pathol 2011; 42: 669-78.

11. Xie Y, Wen X, Jiang Z, Fu HQ, Han H, Dai L. Aquaporin 1 and aquaporin 4 are involved in invasion of lung cancer cells. Clin Lab 2012; 58: 75-80.

12. Wei $X$, Dong J. Aquaporin 1 promotes the proliferation and migration of lung cancer cell in vitro. Oncol Rep 2015; 34: 1440-8.

13. Sato M, Shames DS, Hasegawa Y. Emerging evidence of epithelialto-mesenchymal transition in lung carcinogenesis. Respirology 2012; 17: 1048-59.

14. Kase S, Sugio K, Yamazaki K, Okamoto T, Yano T, Sugimachi K. Expression of E-cadherin and beta-catenin in human non-small cell lung cancer and the clinical significance. Clin Cancer Res 2000; 6: 4789-96. 
15. Shi Y, Wu H, Zhang M, Ding L, Meng F, Fan X. Expression of the epithelial-mesenchymal transition-related proteins and their clinical significance in lung adenocarcinoma. Diagn Pathol 2013; 8: 89.

16. Yoshida T, Hojo S, Sekine $S$, et al. Expression of aquaporin- 1 is a poor prognostic factor for stage II and III colon cancer. Mol Clin Oncol 2013; 1: 953-8.

17. Jiang Y. Aquaporin- 1 activity of plasma membrane affects HT20 colon cancer cell migration. IUBMB Life 2009; 61: 1001-9.

18. Yin T, Yu S, Xiao L, Zhang J, Liu C, Lu Y. Correlation between the expression of aquaporin 1 and hypoxia-inducible factor 1 in breast cancer tissues. J Huazhong Univ Sci Technolog Med Sci 2008; 28: 346-8.

19. Travis WD, Brambilla E, Noguchi M, et al. International Association for the Study of Lung Cancer/American Thoracic Society/European Respiratory Society international multidisciplinary classification of lung adenocarcinoma. J Thorac Oncol 2011; 6: 244-85.

20. Travis WD, Brambilla E, Burke AP, Marx A, Nicholson AG. WHO classification of tumours of the lung, pleura, thymus and heart. Lyon: IARC Press, 2015.

21. Li XQ, Yang XL, Zhang G, et al. Nuclear beta-catenin accumulation is associated with increased expression of Nanog protein and predicts poor prognosis of non-small cell lung cancer. J Transl Med 2013; 11: 114 .

22. Kim H, Yoo SB, Sun P, et al. Alteration of the E-cadherin/betacatenin complex is an independent poor prognostic factor in lung adenocarcinoma. Korean J Pathol 2013; 47: 44-51.

23. Chung JH, Choe G, Jheon S, et al. Epidermal growth factor receptor mutation and pathologic-radiologic correlation between multiple lung nodules with ground-glass opacity differentiates multicentric origin from intrapulmonary spread. J Thorac Oncol 2009; 4: 1490-5.

24. Deb P, Pal S, Dutta V, Boruah D, Chandran VM, Bhatoe HS. Correlation of expression pattern of aquaporin-1 in primary central nervous system tumors with tumor type, grade, proliferation, microvessel density, contrast-enhancement and perilesional edema. J Cancer Res Ther 2012; 8: 571-7.

25. Bartis D, Mise N, Mahida RY, Eickelberg O, Thickett DR. Epithelialmesenchymal transition in lung development and disease: does it exist and is it important? Thorax 2014; 69: 760-5.

26. Monzani E, Bazzotti R, Perego C, La Porta CA. AQP1 is not only a water channel: it contributes to cell migration through Lin7/betacatenin. PLoS One 2009; 4: e6167.

27. Hu J, Verkman AS. Increased migration and metastatic potential of tumor cells expressing aquaporin water channels. FASEB J 2006; 20: $1892-4$.

28. Johnson $\mathrm{MD}, \mathrm{O}^{\prime} \mathrm{C}$ nnell $\mathrm{M}$. Na-K-2Cl cotransporter and aquaporin 1 in arachnoid granulations, meningiomas, and meningiomas invading dura. Hum Pathol 2013; 44: 1118-24.

29. Oshio K, Binder DK, Liang Y, et al. Expression of the aquaporin-1 water channel in human glial tumors. Neurosurgery 2005; 56:375-81. 